\title{
Putting a Population Health Lens to Multimorbidity in Ontario
}

Laura Rosella and Kathy Kornas

\begin{abstract}
Almost all Ontarians die with multimorbidity, and most accumulate more than five conditions over their lifetime. Our health system is still largely focused on specialties and treating one disease at a time - an approach that is incompatible with the healthcare needs of patients with multiple and often complex chronic conditions. This burden requires a health system that recognizes that patients will more likely live and die with multiple chronic conditions than not (i.e., multimorbidity management versus specialized care). There are important and meaningful differences in the types and numbers of conditions that patients die with. In particular, increases in the most preventable conditions are greater among the most deprived members of our society. To address the worrying trends seen here, chronic disease
\end{abstract}

prevention - not only management - must be a priority, with a strong focus on health equity. Chronic disease prevention and a strong focus on equity are signatures of a population health approach. This work echoes calls for a stronger emphasis on population health in the health system.

\section{The Issue}

The co-occurrence of multiple chronic conditions - known as multimorbidity - is now the typical trajectory for patients in a health system. In high-income countries, the burden of chronic diseases on healthcare systems is increasingly exacerbated by a growing proportion of the population having multiple chronic conditions (Fortin et al. 2012; McPhail 2016; Violan et al. 2014). For instance, in 2009, it was estimated that a quarter of

FIGURE 1.

Accumulation of chronic conditions in Ontario (1994-2013), number of adult deaths $=1,634,366$

1994

$(N=74,227)$

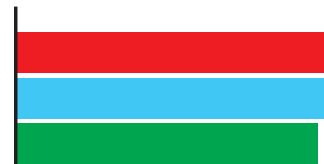

2004

$(N=80,725)$
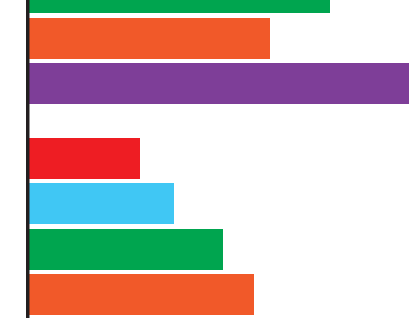

2013

$(N=91,312)$

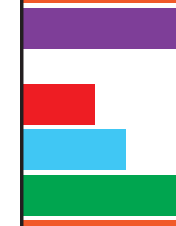

No multimorbidity (0-1 condition)

2 conditions

3 conditions

4 conditions

$5+$ conditions

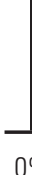

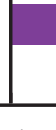

$20 \%$

$40 \%$

$60 \%$

Percentage of decedents with degree of multimorbidity 
Ontario's population lives with multimorbidity (Koné Pefoyo et al. 2015). In addition, $>65 \%$ of Ontario residents die with five or more chronic conditions, an increase from 39\% from 1994 to 2013 (Rosella et al. 2018) (Figure 1).

Multimorbidity is not characterized by any dominant combination of co-occurring conditions (Koné Pefoyo et al. 2015), which presents challenges for decision makers working to allocate health resources in health systems that traditionally use disease-specific approaches (Barnett et al. 2012). Most health systems are oriented around specialty care and are not set up to deal with typical patients, who will have more than one condition, particularly as they age (Ryan et al. 2018). Patients who have multiple and often complex conditions require a more holistic and integrated healthcare process, especially given that they are more likely to receive care from different types of healthcare providers (Kastner et al. 2018; Tricco et al. 2014). There have been several calls to address the silos in medical care delivery to provide more comprehensive care to patients (Albreht et al. 2016). Those calls need to be addressed to improve patient experience and efficiency of healthcare delivery (Leijten et al. 2018). However, multimorbidity is more than a health system organization issue - it is also about prevention and equity. The multimorbidity burden will not meaningfully change if stopped at improving chronic disease management. Efforts must be made to prevent chronic diseases and address persistent health inequities.

The burden of multimorbidity is not experienced equally across the population. A recent study of all deaths in Ontario, which examined multimorbidity accumulated over the life course, found socioeconomic differences in the burden and nature of multimorbidity (Rosella et al. 2018) (Figure 2). The nature of the disparity and conditions seen in Figure 2 are also telling. Chronic obstructive pulmonary disease (COPD) and diabetes are the two conditions that are the most prevalent in low-income areas. These conditions are not an inevitability of aging but rather preventable conditions influenced by known modifiable risk factors, such as smoking and obesity, and upstream determinants rooted in social disadvantage. Several subsequent studies have confirmed that the patterning of chronic conditions and multimorbidity in the population was more prevalent among those living in the most deprived areas (Ryan et al. 2018, Tetzlaff et al. 2018), which translates into disparities in the population and higher healthcare costs (Thavorn et al. 2017). A comprehensive response to the multimorbidity burden will inherently require a population health approach that takes action on the upstream causes of inequities that put people at risk for certain chronic conditions and onto a multimorbidity trajectory.

\section{FIGURE 2.}

Age-adjusted differences in the presence of chronic conditions at the time of death across area income quintile 1 (lowest) and quintile 5 (highest) in Ontario (1994-2013; number of adult deaths = 1,634,366)

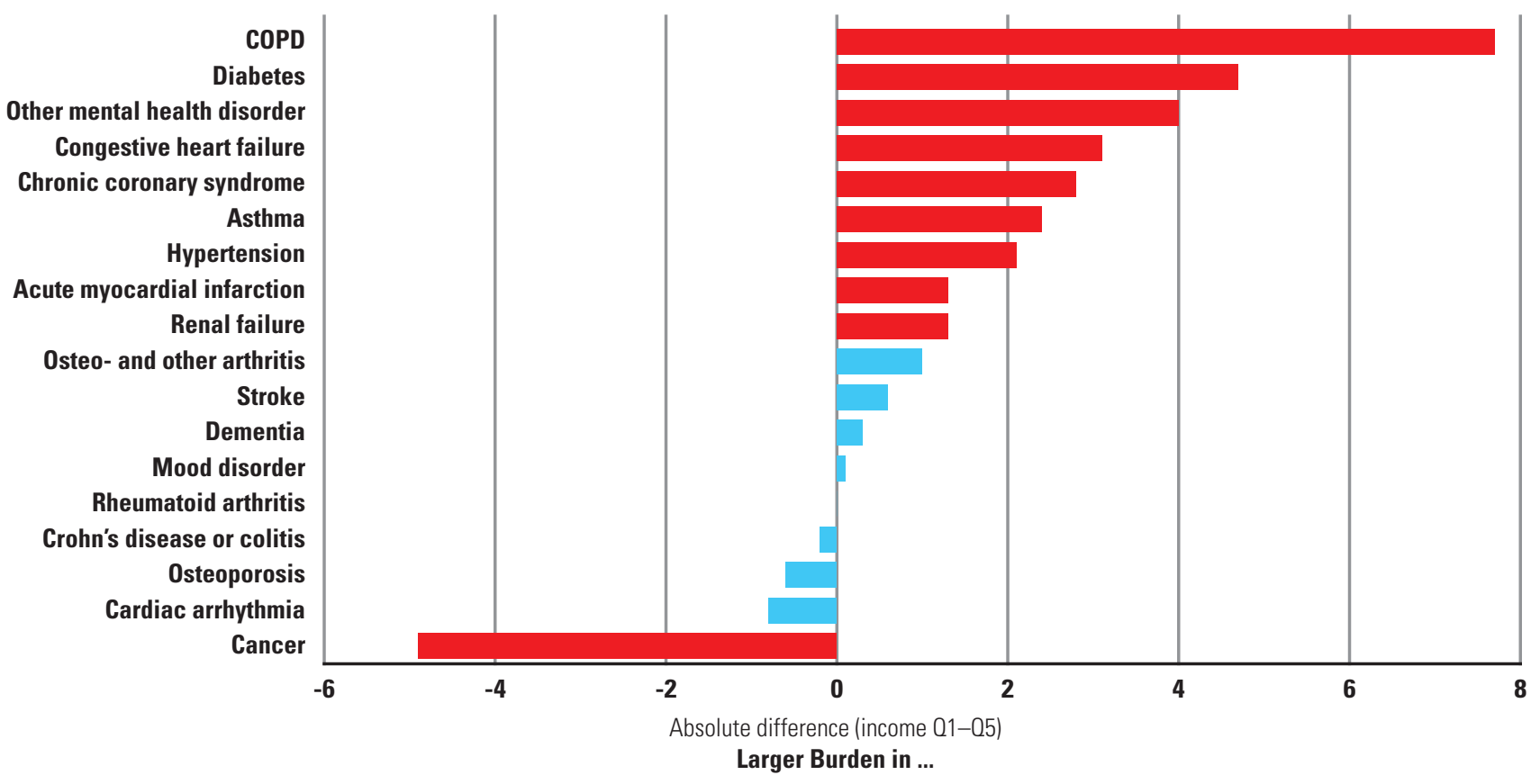

High-income neighbourhoods $\longrightarrow \longrightarrow$ Low-income Neighbourhoods 


\section{Moving Forward}

To address the growing burden of multimorbidity in the population, several directions should be pursued.

\section{Upstream actions for the prevention of chronic diseases}

From the perspective of multimorbidity, unless we start investing in and reorienting our health system to prevent the chronic conditions from accumulating in the first place, we have little hope of changing the trends. Most health systems are still oriented toward a more reactive healthcare approach after the diagnosis of disease. Yet many of the conditions that people are treated for over the life course, including hypertension, diabetes, COPD and certain cancers, are largely preventable through policy and public health efforts that target risky behaviours and socioeconomic factors (Bauer et al. 2014; Oliver 2018). Despite repeated calls for increased focus on investments in housing, food environments, built spaces and other upstream health determinants (Manuel et al. 2009; Williams et al. 2008) and recognition of the value of disease prevention across the political spectrum, chronic diseases continue to rise, with little evidence of tangible progress. Awareness and acceptance are not the issue. Society and many governments generally accept that it is better to prevent than to cure or treat. Furthermore, there have been several economic arguments for a strong return on investment for prevention (Masters et al. 2017). The question remains: Why have there not been more significant efforts at targeting chronic disease prevention? Perhaps it is the inertia of the health system or the lack of funding that results in the response of treating disease versus proactively mitigating its burden. The reality is that it is more complicated than funding or the will to change. Effective and impactful prevention strategies require multistakeholder and multisector collaboration, making such approaches difficult to initiate and sustain.

\section{Addressing inequities in the multimorbidity burden}

Meaningful reductions in multimorbidity will only be realized through concerted efforts focused on addressing inequities. It has been shown that those living in the most deprived areas experienced higher levels of multimorbidity, particularly for chronic conditions that were preventable (Lund Jensen et al. 2017; Mondor et al. 2018; Rosella et al. 2018; Thavorn et al. 2017). There is a sector of the health system in Ontario that indeed focuses heavily on both prevention and equity - the public health sector. The public health and healthcare sectors are still largely siloed for historical, funding and structural reasons. There have been increasing calls to better align the healthcare and public health sectors. One response is the Patients First Act, 2016, in Ontario, which requires that public health units and the health service regions (i.e., Local Health Integration Networks) work together on population health strategies. This and other recommendations all point in a single direction: for meaningful prevention and focus on equity to occur, the healthcare and public health sectors of our system must be enabled to work together in a meaningful way. Elevating the role of public health professionals in the health system will enable the population health focus that is needed to reorient the system. There has been more effort than ever to work together. The impact of these improved relationships has yet to be seen in Ontario, but the strong orientation and motivation toward population health are a positive sign.

\section{A holistic healthcare approach for those with multimorbidity}

For those who experience multimorbidity, the system should be oriented toward a person-centred perspective that recognizes the common trajectory of multimorbidity and in which healthcare and chronic disease management for such patients is approached from the patient perspective. This aligns with many positive initiatives being implemented in the health system, including a focus on patient-centred care and supports to better manage complex patients. What can be added to the positive progress in this area is a recognition of the person's broader circumstances. This means that sometimes patients with multimorbidity will need to receive not only integrated multispecialty care but also social support, such as housing support, which can help them cope with their multiple conditions.

\section{Conclusions}

The majority of patients in the health system are likely to struggle with multiple chronic disease diagnoses by the end of their life. This is difficult for patients, their families and the health system that must respond. Furthermore, certain segments of society, including the economically disadvantaged, will bear this burden more than others. By taking a population health lens to multimorbidity, efforts to prevent chronic disease development and reduce inequities will translate into a healthier population and reduced strains on the health system. The gains needed to reduce the burden and disparities of multimorbidity will require an upstream, large-scale and coordinated action across jurisdictions and among health and social sectors. HQ

\section{References}

Albreht, T., M. Dyakova, F.G. Schellevis and S. Van den Broucke. 2016. "Many Diseases, One Model of Care?" Journal of Comorbidity 6(1): 12-20. doi:10.15256/joc.2016.6.73.

Barnett, K., S.W. Mercer, M. Norbury, G. Watt, S. Wyke and B. Guthrie. 2012. "Epidemiology of Multimorbidity and Implications for Health Care, Research, and Medical Education: A Cross-Sectional Study." Lancet 380(9836): 37-43. doi:10.1016/S0140-6736(12)60240-2. 
Bauer, U.E., P.A. Briss, R.A. Goodman and B.A. Bowman. 2014. "Prevention of Chronic Disease in the 21st Century: Elimination of the Leading Preventable Causes of Premature Death and Disability in the USA." Lancet 384(9937): 45-52. doi:10.1016/S01406736(14)60648-6.

Fortin, M., M. Stewart, M.E. Poitras, J. Almirall and H. Maddocks. 2012. "A Systematic Review of Prevalence Studies on Multimorbidity: Toward a More Uniform Methodology." Annals of Family Medicine 10(2): 142-51. doi:10.1370/afm.1337.

Kastner, M., R. Cardoso, Y. Lai, V. Treister, J.S. Hamid, L. Hayden et al. 2018. "Effectiveness of Interventions for Managing Multiple High-Burden Chronic Diseases in Older Adults: A Systematic Review and Meta-Analysis." CMAJ 190(34): E1004-12. doi:10.1503/ cmaj.171391.

Koné Pefoyo, A.J., S.E. Bronskill, A. Gruneir, A. Calzavara, K. Thavorn, Y. Petrosyan et al. 2015. "The Increasing Burden and Complexity of Multimorbidity." BMC Public Health 15: 415. doi:10.1186/s12889015-1733-2.

Leijten, F.R.M., M. Hoedemakers, V. Struckmann, M. Kraus, S. Cheraghi-Sohi, A. Zemplényi et al. 2018. "Defining Good Health and Care from the Perspective of Persons with Multimorbidity: Results from a Qualitative Study of Focus Groups in Eight European Countries." BMJ Open 8(8): e021072. doi:10.1136/bmjopen-2017-021072.

Lund Jensen, N., H.S. Pedersen, M. Vestergaard, S.W. Mercer, C. Glümer and A. Prior. 2017. "The Impact of Socioeconomic Status and Multimorbidity on Mortality: A Population-Based Cohort Study." Clinical Epidemiology 9: 279-89. doi:10.2147/CLEP.S129415.

Manuel, D.G., M.I. Creatore, L.C.A. Rosella and D.A. Henry. 2009. What Does It Take to Make a Healthy Province? A Benchmark Study of Jurisdictions in Canada and around the World with the Highest Levels of Health and the Best Health Behaviours. Toronto, ON: Institute for Clinical Evaluative Sciences. Retrieved September 25, 2018. <https:// www.ices.on.ca/Publications/Atlases-and-Reports/2009/What-does-ittake-to-make-a-healthy-provinces.

Masters, R., E. Anwar, B. Collins, R. Cookson and S. Capewell. 2017. "Return on Investment of Public Health Interventions: A Systematic Review." Journal of Epidemiology and Community Health 71(8): 827-34. doi:10.1136/jech-2016-208141.

McPhail, S.M. 2016. "Multimorbidity in Chronic Disease: Impact on Health Care Resources and Costs." Risk Management and Healthcare Policy 9: 143-56. doi:10.2147/RMHP.S97248.

Mondor, L., D. Cohen, A.I. Khan and W.P. Wodchis. 2018. "Income Inequalities in Multimorbidity Prevalence in Ontario, Canada: A Decomposition Analysis of Linked Survey and Health Administrative Data." International Journal for Equity in Health 17(1): 90. doi:10.1186/ s12939-018-0800-6.

Oliver, D. 2018. "David Oliver: Getting Serious about Prevention." BMJ 360: k583. doi:10.1136/bmj.k583.
Rosella, L., K. Kornas, A. Huang, C. Bornbaum, D. Henry and W.P. Wodchis. 2018. "Accumulation of Chronic Conditions at the Time of Death Increased in Ontario from 1994 to 2013." Health Affairs (Millwood) 37(3): 464-72. doi:10.1377/hlthaff.2017.1150.

Ryan, B.L., K.B. Jenkyn, S.Z. Shariff, B. Allen, R.H. Glazier, M. Zwarenstein et al. 2018. "Beyond the Grey Tsunami: A Cross-Sectional Population-Based Study of Multimorbidity in Ontario." Canadian Journal of Public Health 109(5-6): 845-54. doi:10.17269/s41997018-0103-0.

Tetzlaff, J., J. Epping, S. Sperlich, S. Eberhard, J.T. Stahmeyer and S. Geyer. 2018. "Widening Inequalities in Multimorbidity? Time Trends among the Working Population between 2005 and 2015 Based on German Health Insurance Data." International Journal for Equity in Health 17(1): 103. doi:10.1186/s12939-018-0815-z.

Thavorn, K., C.J. Maxwell, A. Gruneir, S.E. Bronskill, Y. Bai, A.J. Koné Pefoyo et al. 2017. "Effect of Socio-demographic Factors on the Association between Multimorbidity and Healthcare Costs: A Population-Based, Retrospective Cohort Study." BMJ Open 7(10): e017264. doi:10.1136/bmjopen-2017-017264.

Tricco, A.C., J. Antony, N.M. Ivers, H.M. Ashoor, P.A. Khan, E. Blondal et al. 2014. "Effectiveness of Quality Improvement Strategies for Coordination of Care to Reduce Use of Health Care Services: A Systematic Review and Meta-Analysis." CMAJ 186(15): E568-78. doi: 10.1503/cmaj.140289.

Violan, C., Q. Foguet-Boreu, G. Flores-Mateo, C. Salisbury, J. Blom, M. Freitag et al. 2014. "Prevalence, Determinants and Patterns of Multimorbidity in Primary Care: A Systematic Review of Observational Studies.” PLoS One 9(7): e102149. doi:10.1371/ journal.pone.0102149.

Williams, D.R., M.V. Costa, A.O. Odunlami and S.A. Mohammed. 2008. "Moving Upstream: How Interventions that Address the Social Determinants of Health Can Improve Health and Reduce Disparities." Journal of Public Health Management and Practice 14(Suppl): S8-17. doi:10.1097/01.PHH.0000338382.36695.42.

\section{About the Authors}

Laura Rosella, $\mathrm{PhD}$, is an associate professor at the Dalla Lana School of Public Health, University of Toronto, and the Canada Research Chair in Population Health Analytics. She can be reached at laura.rosella@utoronto.ca.

Kathy Kornas, MSc, is a research officer and epidemiologist at the Population Health Analytics Laboratory located within the Dalla Lana School of Public Health at the University of Toronto. She can be reached at kathy.kornas@utoronto.ca. 